\title{
Retraction Note to: Changes of Absorptive and Secretory Transporting System of $(1 \rightarrow 3) \beta$-D-glucan Based on Efflux Transporter in Indomethacin-induced Rat
}

\author{
Aiko lida ${ }^{1}$. Shohei Ouchi ${ }^{1}$ Toshio Oda $^{2}$. Jun Aketagawa ${ }^{3} \cdot$ Yasuhiko Ito $^{1} \cdot$ Yusuke Takizawa $^{1} \cdot$ Mikio Tomita $^{1}$. \\ Masahiro Hayashi ${ }^{1}$
}

Published online: 14 November 2018

(c) Springer Nature Switzerland AG 2018

\section{Retraction to: Eur J Drug Metab Pharmacokinet (2015) 40:29-38 https://doi.org/10.1007/ s13318-014-0174-0}

The Editor-in-Chief has retracted this article [1] based on an investigation by the Ministry of Education, Culture, Sports, Science and Technology, Japan, which found that the article contained overlap with a previously published article by Kalitsky-Szirtes J, et al. [2]. All authors agree with the retraction, but the authors do not agree with the wording of the retraction note.

[1] Iida A, Ouchi S, Oda T, et al. Changes of Absorptive and Secretory Transporting System of $(1 \rightarrow 3) \beta$-D-glucan Based on Efflux Transporter in Indomethacin-induced Rat. Eur J Drug Metab Pharmacokinet. 2015; 40(1): 29-38.
[2] Kalitsky-Szirtes J, Shayeganpour A, Brocks DR, et al. Suppression of drug-metabolizing enzymes and efflux transporters in the intestine of endotoxin-treated rats. Drug Metab Dispos. 2004; 32(1): 20-7.

The original article can be found online at https://doi.org/10.1007/ s13318-014-0174-0.

Yusuke Takizawa

takizawa@toyaku.ac.jp

1 Department of Drug Absorption and Pharmacokinetics, School of Pharmacy, Tokyo University of Pharmacy and Life Sciences, 1432-1 Horinouchi, Hachioji, Tokyo 192-0392, Japan

2 Research and Development Department, Research Laboratories, Seikagaku Corporation, 1253 Tateno 3-chome, Higashiyamato, Tokyo 207-0021, Japan

3 Technical Support Group, LAL and Biochemicals Marketing Department, Seikagaku Corporation, 6-1 Marunouchi 1-chome, Chiyoda-ku, Tokyo 101-0042, Japan 